\title{
Transmembrane protein 64 modulates prostate tumor progression by regulating Wnt3a secretion
}

\author{
YEON HEE MOON ${ }^{1 *}$, WONBONG LIM ${ }^{2-4 *}$ and BYUNG-CHUL JEONG ${ }^{2,3}$ \\ ${ }^{1}$ Department of Dental Hygiene, Chodang University, Muangun, Jeollanamdo 58530; ${ }^{2}$ Department of Orthopaedic Surgery; \\ ${ }^{3}$ Laboratory of Orthopaedic Research, Department of Orthopaedic Surgery, Chosun University Hospital, \\ Donggu, Gwangju 61453; ${ }^{4}$ Department of Premedical Program, School of Medicine, Chosun University, \\ Donggu, Gwangju 61452, Republic of Korea
}

Received November 15, 2018; Accepted April 29, 2019

DOI: $10.3892 / \mathrm{ol} .2019 .10324$

\begin{abstract}
Wnt3a is a glycosylated ligand that activates the $\beta$-catenin-dependent signaling pathway. Wnt signaling is also important in the prostate tumor microenvironment, and Wnt proteins secreted by the tumor stroma promote resistance to therapy. Bioactive Wnt3a production requires a number of dedicated factors in the secretory cell, but their coordinated functions are not fully understood. We previously reported transmembrane protein 64 (Tmem64) as a novel regulator of the Wnt/ $\beta$-catenin signaling pathway, which is correlated with $\beta$-catenin regulation. In the present study, the role of Tmem64 in prostate cancer cells was investigated by modulating Wnt3a secretion. Overexpression of Tmem64 inhibited Wnt3a secretion and Lef/Tcf-sensitive transcription. By contrast, a Tmem64 mutation deleting the protein's transmembrane region restored Wnt3a secretion. Notably, Tmem64 protein and mRNA in PC3 cells were significantly overexpressed compared with that observed in LNCaP and DU145 cells. In a mouse metastasis model intracardially injected with PC3 cells, Tmem64 expression was downregulated in the metastatic spine and mandible lesions compared with in the primary injection regions. However, Wnt3a was strongly expressed in the metastatic spine and mandible lesions. Collectively, these findings suggest that Tmem64 is involved in the metastatic progression of prostate cancer cells by regulating Wnt3a secretion.
\end{abstract}

\section{Introduction}

Wnt proteins regulate many stages of development, including patterning of the embryo, initiation of axon guidance, and

Correspondence to: Dr Byung-Chul Jeong, Laboratory of Orthopaedic Research, Department of Orthopaedic Surgery, Chosun University Hospital, 309 Philmundaero, Donggu, Gwangju 61453, Republic of Korea

E-mail: bcfrank@chosun.ac.kr

*Contributed equally

Key words: transmembrane protein 64 , Wnt3a, secretion, prostate cancer, metastasis synaptic formation (1). Wnt signaling plays a major role in bone development and its defects can lead to skeletal diseases such as osteoporosis $(2,3)$. Loss of Wnt leads to tetra-amelia syndrome, a severe congenital defect in which all four limbs fail to form, as well as to craniofacial defects (4). During skeletogenesis, conditional removal of $\beta$-catenin in mesenchymal progenitor cells leads to decreased osteoblast differentiation $(5,6)$. In contrast, constitutive activation of $\beta$-catenin under the same conditions results in dramatically increased bone deposition with reduced osteoclast formation (7). The Wnt/ $\beta$-catenin signaling pathway is activated by binding of a Wnt ligand to Frizzled (Fz), a seven transmembrane protein receptor, which mediates subsequent downstream disruption of the $\beta$-catenin degradation complex (8). This allows $\beta$-catenin to be accumulated in the nucleus, where it binds to members of the $\mathrm{T}$ cell factor, lymphoid enhancer factor (Lef/Tcf)/transcription factor family, and activates gene transcription necessary for the development of multicellular organisms and regulation of adult tissue homeostasis, including growth and renewal of bones (9-11). Wnt proteins are glycosylated in the endoplasmic reticulum and palmitoylated (12). Experiments using tunicamycin (an inhibitor of asparagine-linked glycosylation) demonstrated that Wnt3a is modified with $N$-linked glycans (13). However, Wnt proteins are inefficiently secreted and tend to linger in the endoplasmic reticulum. Although the molecular pathways activated by $\mathrm{Wnt} 3 \mathrm{a} / \beta$-catenin have been extensively studied, as summarized above, the mechanisms underlying Wnt production are unclear.

Transmembrane protein 64 (Tmem64) is a seven transmembrane protein that is localized to the endoplasmic reticulum and modulates the nuclear localization of $\beta$-catenin, resulting in the activation of $\beta$-catenin-mediated transcription (14). We hypothesized that Tmem64 has a role in Wnt3a secretion. Upon investigating this hypothesis, we found that introduction of Tmem64 markedly inhibited the secretion of Wnt3a by triggering its aggregation as an insoluble cellular fraction. A deletion mutation of Tmem64 which deleted a transmembrane region did not affect Wnt3a activity and secretion, suggesting that Tmem64 may participate in the modulation of Wnt3a/ $\beta$-catenin signaling through Wnt3a secretion. Interestingly, Tmem64 was strongly expressed in human prostate cancer cells, especially PC 3 cells. In a metastatic 
mouse model created by the intracardiac injection of PC3 cells, Tmem64 expression was down-regulated in metastatic spine and mandible lesions.

Collectively, these findings suggest that Tmem64 is involved in the metastatic progression of prostate cancer cells. These data expand our understanding of the role of Tmem64 in Wnt secretion and provide further support for the potential of Tmem64 as a therapeutic target against bone metastasis of prostate cancer cells via dysregulation of Wnt signaling.

\section{Materials and methods}

Plasmids. The TOPFlash reporter constructs were kindly provided by Dr Kyung-Keun Kim (Chonnam National University). Mutant constructs of Tmem64 were generated using a Site-Directed Mutagenesis Kit (Invitrogen; Thermo Fisher Scientific, Inc.).

Cell culture and transfection. Human embryonic kidney 293 T cells were cultured in Dulbecco's modified Eagle's medium (Gibco-BRL; Life Technologies, Inc.) supplemented with $10 \%$ fetal bovine serum (FBS), $100 \mathrm{U} / \mathrm{ml}$ penicillin, and $100 \mu \mathrm{g} / \mathrm{ml}$ streptomycin. The DU145, LNCaP and PC3 human prostate cancer cell lines were obtained from the American Type Culture Collection (ATCC) and cultured in RPMI-1640 (Invitrogen; Thermo Fisher Scientific, Inc.) supplemented with 10\% FBS and 1\% penicillin-streptomycin (Life Technologies). Cells were transfected with the indicated amounts of expression plasmids using Lipofectamine 2000 (Invitrogen; Thermo Fisher Scientific, Inc.) according to the manufacturer's instructions. As an internal control, a cytomegalovirus $\beta$-galactosidase (CMV) plasmid was co-transfected in each transfection experiment. Forty-eight hours after transfection, the cells were lysed and assayed with the Dual Luciferase Reporter Assay System (Promega). Luciferase activity was normalized to $\beta$-galactosidase activity.

Western blotting. Cells were harvested in lysis buffer (Cell Signaling Technology) and centrifuged for $15 \mathrm{~min}$ at $4^{\circ} \mathrm{C}$. The Protein concentration in the supernatant was determined using a DC Protein Assay kit (Bio-Rad Laboratories). Cellular proteins were separated by sodium dodecyl sulfate polyacrylamide gel electrophoresis (SDS-PAGE) and transferred to a polyvinylidene difluoride membrane. After blocking in Tris-buffered saline with 5\% milk and $0.1 \%$ Tween-20, the membrane was incubated with primary antibodies for active $\beta$-catenin (anti-ABC, 1:1,000; Merck Millipore), $\beta$-catenin (anti- $\beta$-catenin 1:1,000; Cell Signaling Technology), Tmem64 (anti-Tmem64, 1:1,000; MyBioSource), and $\beta$-actin (anti- $\beta$-actin 1:2,000; Cell Signaling Technology). Signals were visualized using an enhanced chemiluminescence reagent (Santa Cruz Biotechnology) in a LAS-4000 Lumino Image Analyzer system (Fujifilm). The band intensities were quantified using ImageJ (http://rsbweb.nih.gov/ij/) under the Gel Analysis Tool. The intensities for each lane was taken as a ratio of the Tmem64 protein over total protein and then normalized to bands for the control group, whose intensity was set to one.

Conditioned medium. 293T cells were transfected with the Wnt3a vector. Twenty-four hours later, the medium was replaced with a serum-free medium. Samples were collected after another $24 \mathrm{~h}$ and equal volumes were loaded and analyzed by SDS-PAGE.

Luciferase reporter assay. Luciferase activity was measured using the Luciferase Assay System (Promega) and detected using a GENios Plus luminometer (Tecan Group Ltd.). The pCMV- $\beta$-galactosidase (pCMV-gal) expression vector was added to each transfection, and the $\beta$-galactosidase assay was carried out as described previously to normalize transfection efficiency (15).

Quantitative PCR. Total cellular RNA was extracted using the TRIzol-trichloromethane method, and RNA quantity was determined spectrophotometrically. Synthesis of cDNA was performed using the Takara PrimeScript ${ }^{\circledR}$ RT reagent Kit (Takara Bio). Quantitative PCR was performed using the SYBR Premix Ex Taq system (Takara Bio) and using the following primers: Tmem64 forward, 5'-GGCGTGGCTGAG GTGAGAAA-3' and reverse, 5'-ATGAAGCCCACGACGAAG AG-3'; glyceraldehyde-3-phosphate dehydrogenase (GAPDH) forward, 5'-CCAGTCAGCTTCCCGTTCA-3' and reverse, 5'-GAACATCATCCCTGCATCCA-3'. The relative messenger RNA (mRNA) levels were calculated based on the threshold cycle $(\mathrm{Cq})$ values normalized to the $\mathrm{Cq}$ value of $\beta$-actin using the following formula: $2^{-\Delta \mathrm{Cq}}$, where $\Delta \mathrm{Cq}=\mathrm{Cq}_{\text {target gene }}-\mathrm{Cq}_{\beta \text {-actin }}$. All tests were performed in triplicate.

Animals. Five-week-old male athymic nude mice (BL-6/Nu; Orient Bio Co., Ltd.) were housed under controlled light conditions and fed ad libitum. All experimental procedures involving animals were performed in compliance with institutional and government requirements and were approved by the Institutional Animal Care and Use Committee (CIACUC2015-A0032), Chosun University, Gwangju, Korea.

Primary or metastatic tumor-bearing mouse model. Primary tumors were generated by injection of PC 3 cells into the flanks of mice. In addition, intracardiac injections of PC3 cells were administered to examine the ability of tumor cells to metastasize. Male severe combined immunodeficient mice (aged 5-6 weeks) were anesthetized using isoflurane gas. PC3 cells $\left(1 \times 10^{5}\right.$ per mouse) were injected into the flanks of mice or the left cardiac ventricle following a modification of a previously described technique (16). After 4 weeks, mice were sacrificed by intraperitoneal administration of $0.4 \%$ sodium pentobarbital $(1 \mathrm{ml} / \mathrm{kg})$ and tumor-bearing tissues were excised and fixed in cold 10\% buffered formalin (Merck). Bone tissue was decalcified in sodium citrate solution. Decalcified bones were cut at the midpoint and embedded in paraffin blocks. Tissue sections were obtained and stained with hematoxylin and eosin (H\&E), and images were captured using a microscope slide scanner (3DHISTECH Ltd.).

Immunohistochemical analysis of bone specimens. Sections ( $3 \mu \mathrm{M}$ thick) were deparaffinized in three changes of xylene and rehydrated in a graded series of ethanol solutions (ending with distilled water). For antigen retrieval, slides were placed in $0.01 \mathrm{M}$ citrate-buffer $(\mathrm{pH} 6)$ and heated in a steamer for $30 \mathrm{~min}$. Endogenous peroxidases were quenched 
by incubating with $3 \%$ hydrogen peroxide for $20 \mathrm{~min}$ at room temperature. Sections were incubated overnight at $4^{\circ} \mathrm{C}$ with a 1:50 dilution of primary antibody (anti-Tmem64; Cell Signaling Technology). Subsequently, sections were incubated for 30 min with a biotinylated secondary antibody (LSAB; Dako Cytomation), washed in phosphate buffered saline, and incubated for $30 \mathrm{~min}$ with a streptavidin-peroxidase conjugate (LSAB; Dako Cytomation). The reaction was developed for 5 min using 3,3'-diaminobenzidine tetrahydrochloride (Sigma-Aldrich). Slides were briefly counterstained in hematoxylin, dehydrated, and cover slipped. Negative and positive controls were run simultaneously. Positive controls comprised mammary tissue. The slides were captured using a microscope slide scanner (3D-HISTECH Ltd.).

Statistical analyses. All experiments were repeated at least twice, and qualitatively identical results were obtained. Statistical analyses were performed using GraphPad Prism version 5.0 software (GraphPad Software, Inc.). A two-tailed, paired Student's t-test and one-way analysis of variance followed by Sidak's multiple comparison test (unless specifically mentioned otherwise) were used when comparing more than two groups. Results are reported as means \pm standard deviation of triplicate independent experiments. A P-value $<0.05$ was considered statistically significant.

\section{Results}

Tmem64 is involved in Wnt3a secretion. To investigate the relationship between Tmem64 and Wnt3a, we co-transfected the Tmem64 expression vectors with a Wnt3aconstructand analyzed the ability of the transfectants to secrete Wnt3a. Introduction of Tmem64 significantly reduced the secretion of Wnt3a into the culture medium in a dose-dependent manner (Fig. 1A). Next, the transcriptional response to $\mathrm{Wnt} / \beta$-catenin signaling was measured using a Lef/Tcf-responsive promoter driving the luciferase reporter (TOPFlash), which specifically measures the transcriptional activation of $\beta$-catenin using conditioned medium $(\mathrm{CM})$ containing the secreted Wnt3a protein. Treatment of cells with Wnt3a-transfected CM (Wnt3a-CM) stimulated the TOPFlash luciferase activity (Fig. 1B). However, Wnt3a-CM with co-transfection of Tmem64 did not affect the luciferase activity (Fig. 1B). Taken together, these results suggest the involvement of Tmem64 in Wnt3a secretion.

Tmem64 mutation does not affect Wnt3a activity and secretion. Tmem64 was predicted as a seven transmembrane protein by the TMpred transmembrane prediction software. Specifically, transmembrane sites five and six of Tmem64 were predicted as important functional regions. To further examine the role of Tmem64 in Wnt3a secretion, we constructed truncated mutants of Tmem64 by deletion of transmembrane regions (Fig. 2A). We have previously shown that Tmem64 modulates the activation of $\beta$-catenin-mediated transcription (14). Therefore, we first examined the possible control of the transcriptional activity of $\beta$-catenin by Tmem64 mutant constructs. Mutations of Tmem64 showed a marginal effect in the inhibition of transcriptional activity of $\beta$-catenin compared to the wild-type construct. Among others, the Tmem64-M3 construct (deletion of 181-295 region of Tmem64) strongly relieved the inhibitory
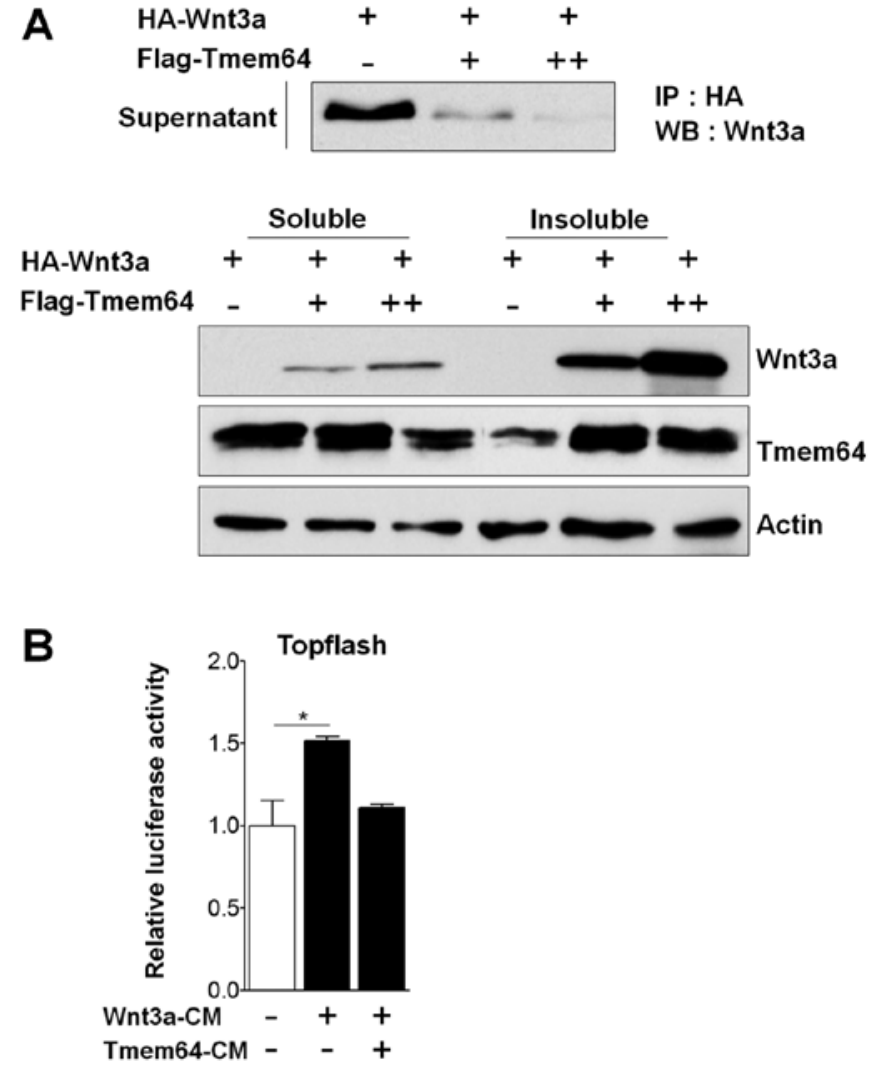

Figure 1. Effects of Tmem64 on Wnt3a secretion. (A) HA-tagged Wnt3a-expression vector was co-transfected with Tmem64 constructs $(+, 100 \mathrm{ng} ;++, 200 \mathrm{ng})$ into $293 \mathrm{~T}$ cells. At $48 \mathrm{~h}$ later, culture medium was collected and cells were harvested. The medium was used for an immunoprecipitation assay using HA-conjugated agarose beads. Western blot analysis was performed using Wnt3a antibody (upper panel). Soluble and insoluble fractions were analyzed by immunoblotting with Wnt3a and Tmem64 antibodies (lower panel). Actin was used as a loading control. (B) 293T cells were transfected with TOPFlash constructs and then treated with the indicated medium. Luciferase values were measured. Data represent the mean \pm standard deviation of samples in triplicates. ${ }^{*} \mathrm{P}<0.05$, as indicated. HA, hemagglutinin; Tmem64, transmembrane protein 64; Rel. Luc, relative luciferase; IP, immunoprecipitation; WB, western blotting; CM, conditioned medium.

effect of Tmem64 (Fig. 2A). Therefore, we further examined the effect of Tmem64-M3 on Wnt3a secretion. Tmem64-M3 did not affect the secretion of Wnt3a in the culture medium and $\beta$-catenin protein levels compared to the wild-type (Fig. 2B). Furthermore, supernatant of Wnt3a-CM with co-transfection of Tmem64-M3 also did not affect luciferase activity of TOPFlash (Fig. 2C), suggesting that Tmem64 mediates the secretion of Wnt3a. In addition, Tmem64 modulates the nuclear localization of $\beta$-catenin, thus inducing $\beta$-catenin-mediated transcriptional activity. We then examined the effect of Tmem64-M3 on $\beta$-catenin nuclear accumulation. The results revealed that $\beta$-catenin was located in the nuclei of the cells treated with Wnt3a (Fig. 2D, upper panel). In contrast, $\beta$-catenin failed to accumulate in the nuclei of the cells expressing Tmem64. Interestingly, the introduction of Tmem64-M3 did not affect $\beta$-catenin nuclear accumulation (Fig. 2D, lower panel). Thus, the nuclear localization of $\beta$-catenin depends on the association of $\beta$-catenin with Tmem64.

Tmem64 is not involved in Wnt3a glycosylation. As Wnt3a is prominently modified with N-linked glycans (12), we 
A Putative transmembrane regions

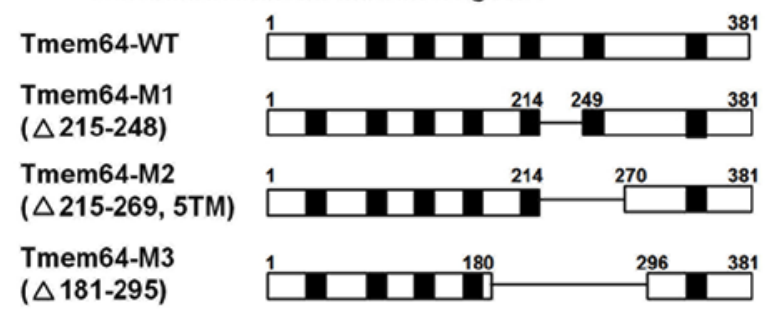

$(\triangle 181-295)$

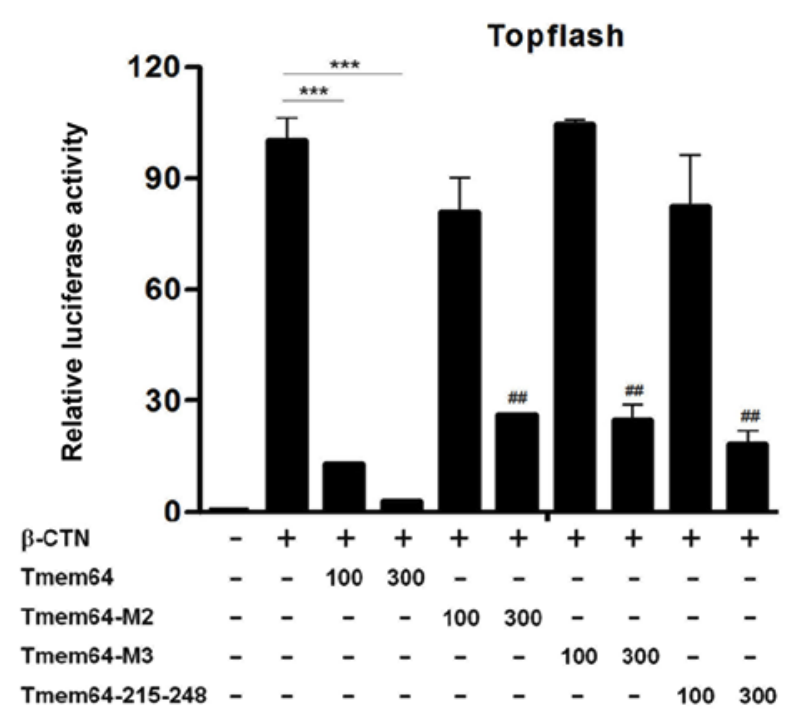

B HA-Wnt3a

$\begin{array}{llll}\text { Flag-Tmem64-WT } & + & + & + \\ \text { Flag-Tmem64-M3 } & - & + & - \\ & & & +\end{array}$
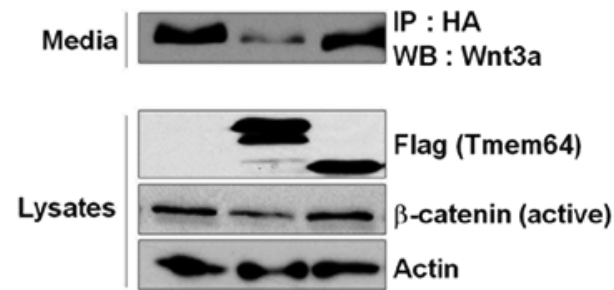

C Topflash

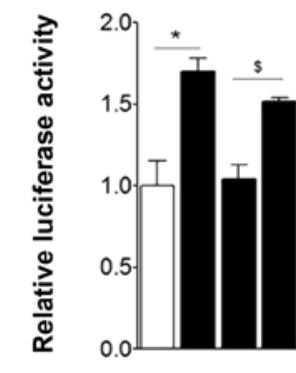

Wnt3a -+++
Tmem64-WT $-\quad+++\mid \begin{aligned} & \text { Conditioned } \\ & \text { medium }\end{aligned}$

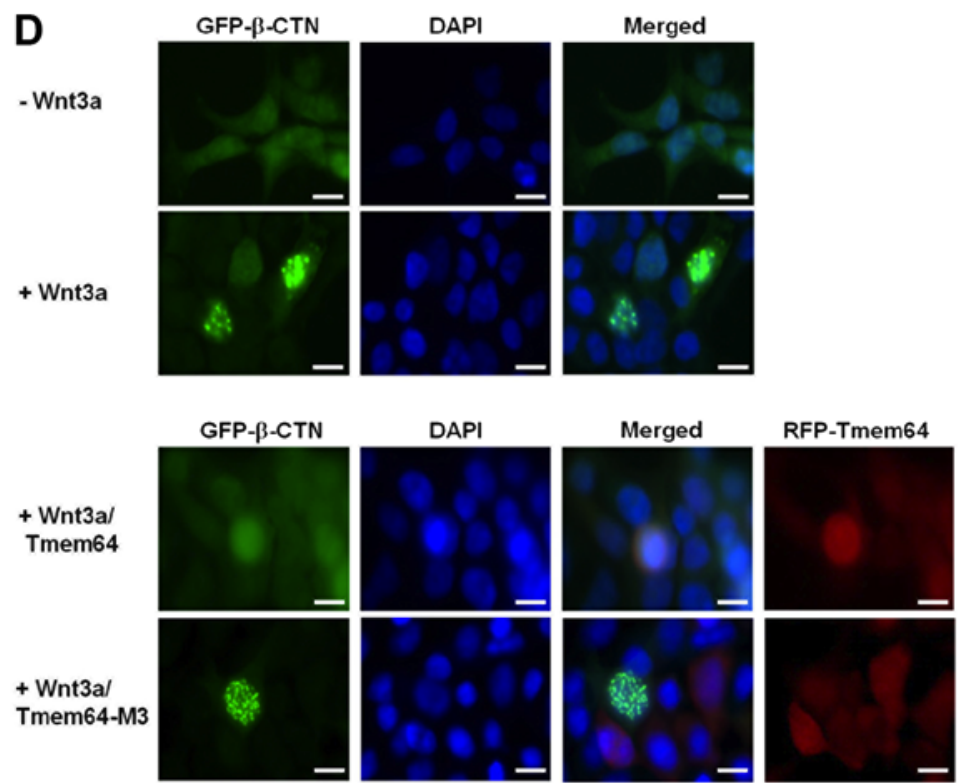

Figure 2. Tmem64 mutant attenuates Wnt/ $\beta$-catenin signaling. (A) Illustration of Tmem64-WT and mutant constructs (upper panel). 293T cells were co-transfected with TOPFlash constructs and the indicated constructs, and luciferase values were measured (lower panel). ${ }^{* * *} \mathrm{P}<0.001$, as indicated; ${ }^{\# \#} \mathrm{P}<0.01$ vs. $\beta$-catenin-treated group. (B) HA-tagged Wnt3a expression construct and Tmem64-WT or Tmem64-M3 were co-transfected into 293T cells. At $48 \mathrm{~h}$ later, the culture medium was collected and cells were harvested. Medium was subjected to an IP assay using HA-conjugated agarose beads. Western blot analysis was performed using a Wnt3a antibody. Lysates were analyzed by immunoblotting with $\beta$-catenin and Tmem64 antibodies (lower panel). Actin was used as a loading control. (C) 293T cells were transfected with TOPFlash constructs and the indicated CM was used as treatment. Luciferase values were measured. Data represent the mean \pm standard deviation of samples in triplicates. ${ }^{*} \mathrm{P}<0.05$ vs. control group; ${ }^{\$} \mathrm{P}<0.05$ vs. Tmem64-WT group. (D) $293 \mathrm{~T}$ cells were transfected with $\beta$-catenin (GFP) and/or Tmem64 (RFP) expression vectors and stimulated with Wnt3a for $12 \mathrm{~h}$. Cells were stained for $\beta$-catenin (green immunofluorescence) and Tmem64 (red immunofluorescence) and counterstained with DAPI (blue). Scale bars, $20 \mu \mathrm{m}$. WT, wild-type; IP, immunoprecipitation; WB, western blotting; HA, hemagglutinin; Tmem64, transmembrane protein 64; CM, conditioned medium; GFP, green fluorescent protein; DAPI, 4',6-diamidino-2-phenylindole; $\beta$-CTN, $\beta$-catenin.

examined whether the glycosylation status of Wnt3a is modulated by Tmem64. Tmem64 overexpression was confirmed by western blotting (data not shown). When Wnt3a was completely de-glycosylated with $\mathrm{N}$-glycosidase $\mathrm{F}$, a peptide 
that cleaves asparagine-linked glycans, a single rapidly migrating $42 \mathrm{kDa}$ band of Wnt3a was observed (Fig. 3A). Moreover, Tmem64-modified Wnt3a still migrated at $42 \mathrm{kDa}$, revealing that Tmem64 modification does not involve an $\mathrm{N}$-glycan. In addition, a series of experiments using tunicamycin, which inhibits the synthesis of N-linked glycoprotein, demonstrated that Tmem64 did not affect Wnt3a expression with asparagine-linked glycans (Fig. 3B).

Involvement of Tmem64 in prostate cancer progression. Dysregulation of $\beta$-catenin is associated with the development of a number of types of cancers, including prostate cancer (17). In addition, inhibition of STMN1, which is overexpressed in aggressive prostate cancer, significantly downregulates the expression of Tmem64 in prostate cancer cells (18). Therefore, we sought to determine the possible involvement of Tmem64 in prostate cancer cell lines. To explore this possibility, we first examined the endogenous expression of Tmem64 in various prostate cancer cell lines derived from human bone metastases (PC3), lymph node metastases (LNCaP), and brain metastases (DU145). Interestingly, PC3 cells expressed markedly higher mRNA levels of Tmem64, but lower levels in both LNCaP and DU145 cells (Fig. 4A). Consistently, western blotting experiments showed that these changes also occurred at the protein level in these cells (Fig. 4B). We next examined whether Tmem64 could participate in the progression of prostate cancer following subcutaneous implantation of cells or in the metastasis of prostate cancer cells to bone in vivo by intracardiac injection of PC3 tumor cells, a well-established model of experimental bone metastasis (19). An apparent margin between tumor cells and other stromal cells was observed in PC3-inoculated mice (Fig. 4C). Tumor cells were well-distinguished from stromal and other functional cells by the presence of nuclear atypia with prominent nucleoli, increased nuclear size, and reduced cytoplasmic-to-nuclear ratio. In case of mandibular metastatic tumor generated by PC3 inoculation, the tumor cells showed small irregular acinar configuration and an infiltrative growth pattern, as revealed by H\&E staining.

In addition, expression patterns of Tmem64 in serial tissue samples of primary and mandibular metastatic tumors formed upon PC3-cell inoculation were determined. A prominent Tmem64 signal was observed in the PC3 tumor mass that later developed in a primary tumor following subcutaneous injection of cells. However, the expression of Tmem64 was significantly down-regulated in mandibular and bone metastatic tumors generated by PC3 cell inoculation (Fig. 4D, upper panel). In contrast, lower Wnt 3 a expression was observed in the PC3-inoculated primary tumor masses and prominent expression was observed in metastatic and mandibular metastatic tumors generated by PC3 inoculation (Fig. 4D, lower panel). Taken together, these results implicate Tmem64 in prostate tumor progression via its modulation of Wnt3a secretion.

\section{Discussion}

Wnt signaling is important in the prostate tumor microenvironment, where Wnt proteins secreted by the tumor stroma promote resistance to therapy (20). Wnt3a has a seminal role in prostate cancer invasion and metastasis (21). Intracellular
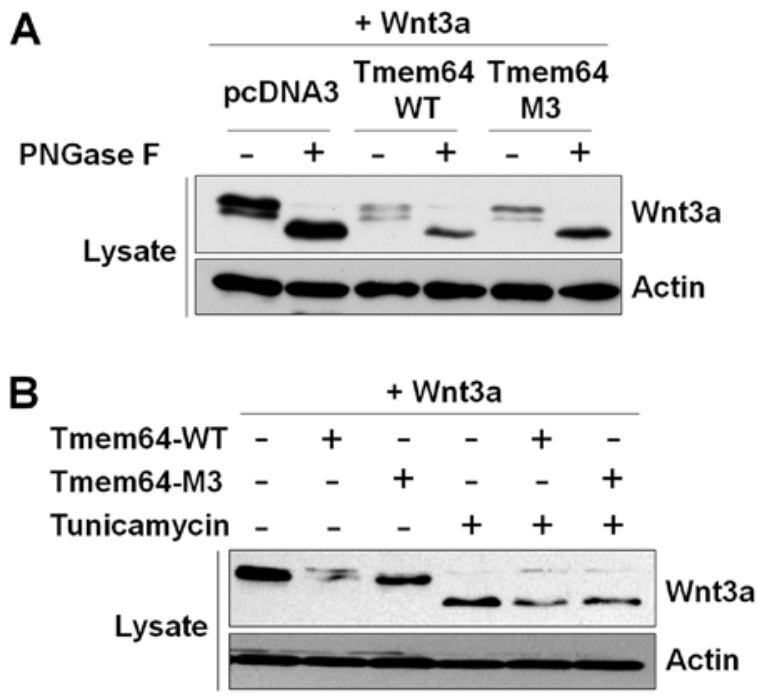

Figure 3. Tmem64 is not associated with the glycosylation of Wnt3a. (A) $293 \mathrm{~T}$ cells were transfected with Tmem64 constructs and then treated with DMSO or PNGaseF for $14 \mathrm{~h}$, as indicated. Glycosylation was assessed by immunoblotting. Actin was used as a loading control. PNGaseF-mediated deglycosylation of Tmem64-modified Wnt3a did not change its migration. (B) 293T cells transfected with Tmem64 and then treated with DMSO or tunicamycin for $7 \mathrm{~h}$, as indicated, following glycosylation were assessed by immunoblotting. Actin was used as a loading control. Tmem64, transmembrane protein 64; WT, wild-type.

accumulation of $\beta$-catenin in response to $\mathrm{Wnt} 3 \mathrm{a}$ is a hallmark of the canonical Wnt signaling pathway and can be used as a reliable marker of Wnt3a signaling activity. We previously showed that Tmem64 modulates the Wnt signaling pathway by association with $\beta$-catenin to decrease the protein level and nuclear localization of the latter (22). To assess the effect of Tmem64 overexpression or inhibition on the Wnt/ $\beta$-catenin signaling pathway, we utilized a Lef/Tcf reporter that can be activated in response to Wnt3a or transfection with constitutively active Wnt signaling components. Overexpression of Tmem64 inhibited $\beta$-catenin accumulation in response to Wnt3a. This result is consistent with the transcriptional readout and is suggestive of negative modulation of $\mathrm{Wnt} / \beta$-catenin signaling by Tmem64.

Several studies have demonstrated that Wnt signaling is positively correlated with prostate cancer progression, with a direct role reported in the induction of bone metastasis $(23,24)$. The expression of $\beta$-catenin in bone metastases appears down-regulated compared to the expression in corresponding primary tumors in patients with untreated prostate cancer (25). Aberrant mutations in the components of Wnt signaling have been linked to multiple growth-related pathologies, particularly to cancer metastasis (26). Several recent studies reported that aberrantly activated $\mathrm{Wnt} / \beta$-catenin signaling may induce tumor formation and progression $(27,28)$. $\beta$-Catenin is a multifunctional transcription factor that is involved in the Wnt signaling pathway and serves an important role in oncogenesis in combination with $\mathrm{T}$ cell factor and protein kinase D1 (29). The dysregulation of $\beta$-catenin is associated with development of several types of cancer, including prostate cancer (17), and the shuttling of $\beta$-catenin between the cytoplasm and the nucleus is pivotal for its pro- or anti-tumor function $(17,30)$. Therefore, a study focusing on the regulation of $\beta$-catenin 


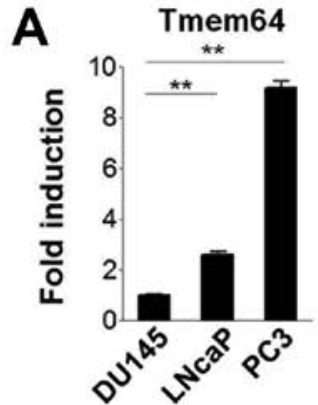

B

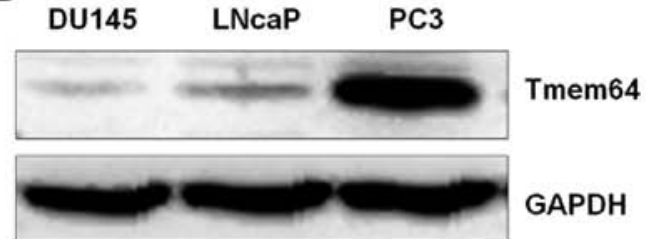

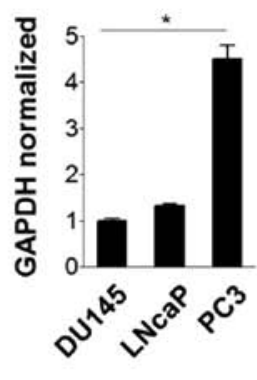

C
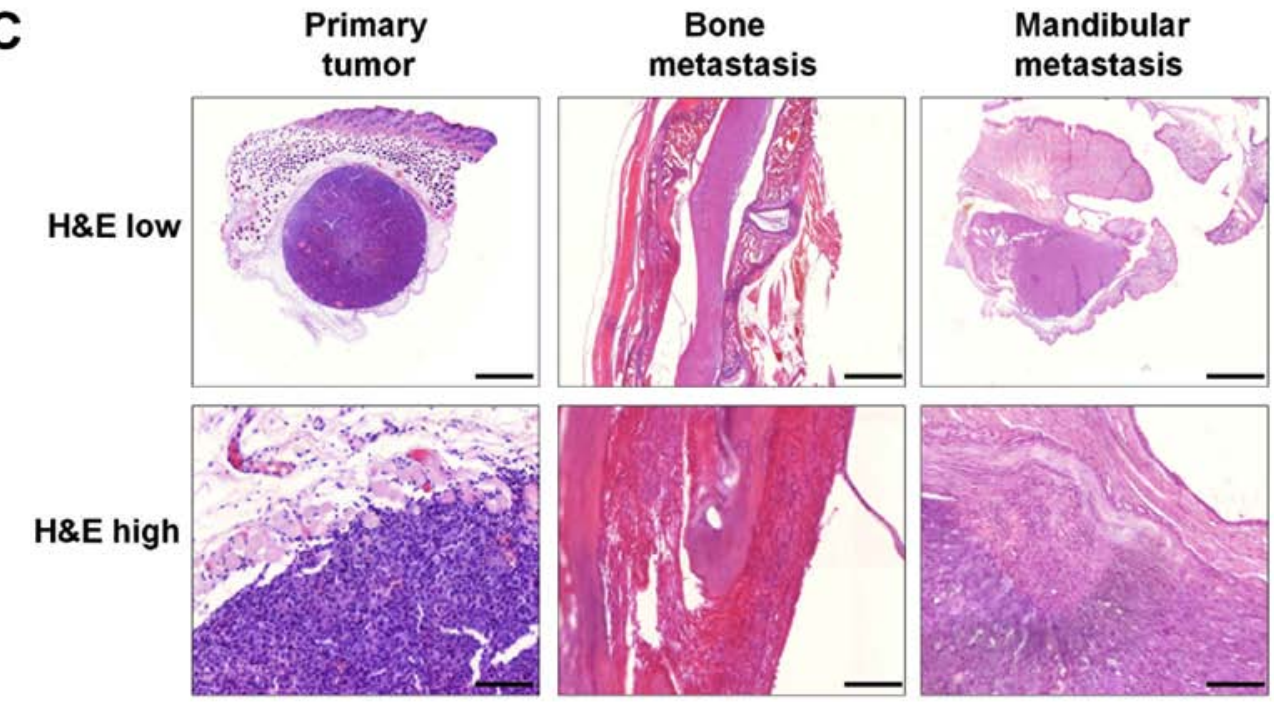

D
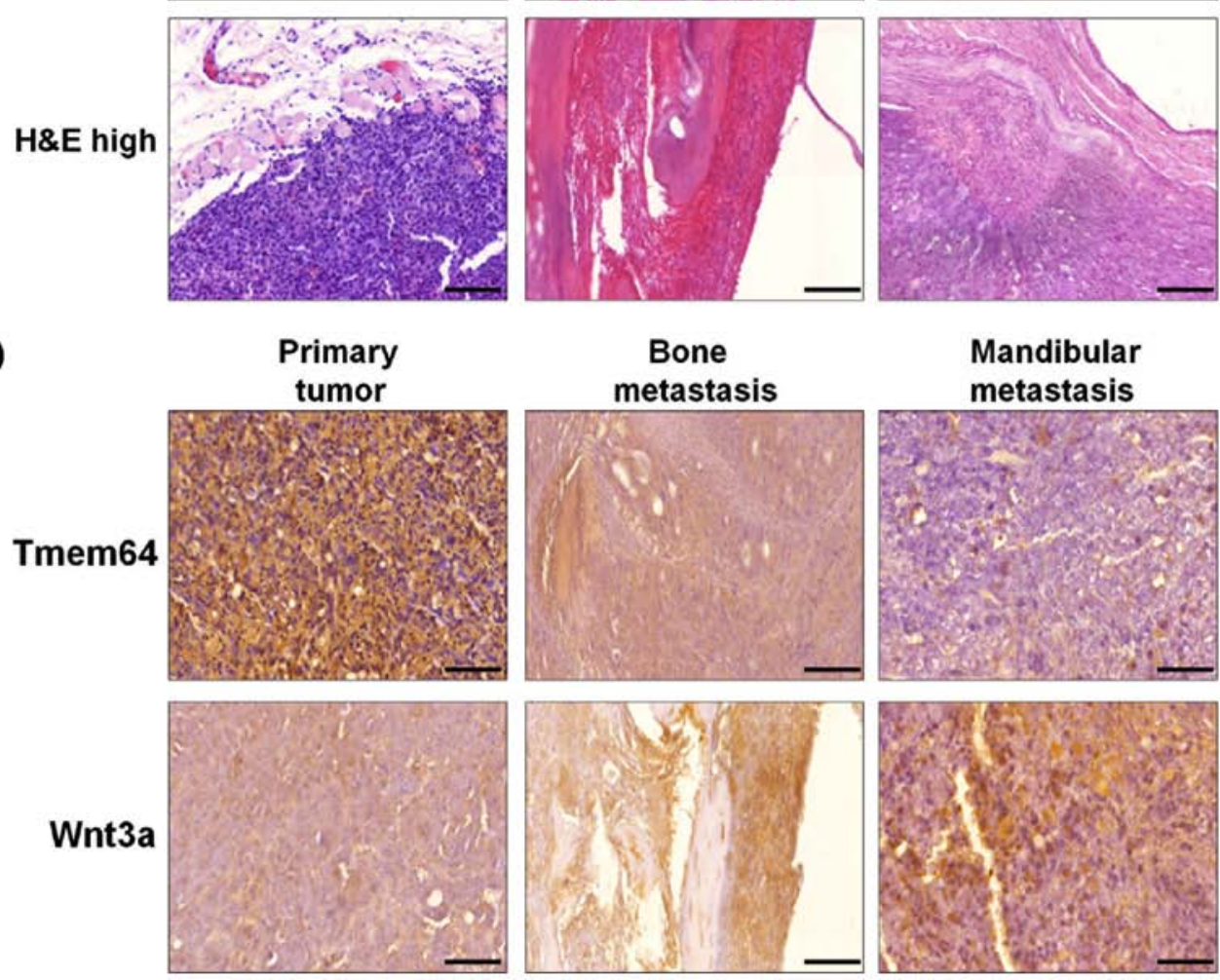

Figure 4. Tmem64 expression in prostate cancer cells and bone metastatic progression. (A) Total RNA was isolated from the indicated cell lysates as described and the mRNA levels of Tmem64 were assessed by reverse transcription-quantitative polymerase chain reaction. Data are presented as the mean \pm standard deviation of samples in triplicate. (B) Protein levels of Tmem64 in various prostate cancers were measured by immunoblotting (left panel). GAPDH was used as the loading control. A representative image of three independent experiments is shown. The bar graph was generated by quantifying band intensities from three independent experiments using ImageJ and normalizing the intensity of the bands to that of the total protein, which was set to one (right panel). ${ }^{*} \mathrm{P}<0.05$ and ${ }^{* *} \mathrm{P}<0.01$, as indicated (C) Representative histological sections of tissues bearing subcutaneous tumors generated by PC 3 inoculation, and tumors generated after intracardiac injection in the mandibular and spine are shown at both low magnification (magnification, x10; scale bar, 2,000 $\mu \mathrm{m}$, upper panel) and high magnification (magnification, x40; scale bar, $100 \mu \mathrm{m}$, lower panel). (D) Immunohistochemical staining of Tmem64 and Wnt3a in the subcutaneous primary, mandibular and spinal tumors generated by PC 3 cells are shown. Positive staining in the subcutaneous nuclei and cytoplasm of tumors (magnification, $\mathrm{x} 400$; scale bar, $50 \mu \mathrm{m}$ ). Tmem64, transmembrane protein 64; H\&E, hematoxylin and eosin.

would highlight the pathological mechanism underlying prostate cancer and suggest novel treatment targets. The results of the present study revealed that Tmem64 decreases the expression of $\beta$-catenin and further support the potential application of Tmem64 as a therapeutic target in prostate cancer.
PC3 cells showed stronger expression of Tmem64 in vitro than the other prostate cancer cell lines. The expression was prominent in the tumor masses that developed following the subcutaneous injection of $\mathrm{PC} 3$ cells. The intracardiac injection in mice introduced the tumor cells directly into the circulation, 
thus facilitating the survival and growth of prostate cancer cells in the other organs (31). We used this mouse model to evaluate the effect of Tmem64 in metastatic prostate cancer. Elevated Tmem64 expression levels were negatively correlated with mandibular and spine metastasis after intracardiac injection. Together, our findings implicate Tmem64 in the metastasis of prostate cancer to the bone.

Bone metastasis of $\mathrm{PCa}$ requires a series of specific interactions between the cancer and host cells, such as bone marrow stromal cells (BMSCs), at the metastatic sites (32). Cell-cell interactions between PCa cells and cells in the bone microenvironment are important and contribute to metastatic cell behavior. This process results in the formation of osteosclerotic lesions filled with metastatic prostate cancer cells. We found that Tmem64 was expressed in prostate cancer cells and prostate cancer-induced metastatic bone lesions. However, our study has some limitations that must be mentioned. The expression level of Tmem64 in bone metastases of prostate cancer was not measured, and the role of Tmem64 in osteoblastic lesions induced by prostate cancer was not assessed. The membrane protein Tmem64 has been reported to restrain bone degradation and promote bone formation in knockout mice (22). In addition, Tmem64 also inhibited osteoblast differentiation and stimulated adipogenesis through the Wnt/ $\beta$-catenin signaling pathway (14). Therefore, Tmem64 is probably involved in the formation of osteosclerotic lesions by regulating $\beta$-catenin production. How Tmem64 participates in this metastatic bone erosion process remains unclear. One possibility is that Tmem64 decreases the potential of prostate cancer to metastasize to bone by modulating the secretion of Wnt3a or expression of $\beta$-catenin. If so, primary cancers with high Tmem64 expression levels would show a poorer prognosis. In addition, mRNA and protein analyses have indicated that Tmem64 expression is a significant independent predictor of poorer prognosis in prostate cancer (14). In terms of clinical applications, Tmem64 may be a candidate marker for the diagnosis of bone metastatic progression in prostate cancer patients. Further studies involving larger number of mice or patient cohorts would be needed to verify this potential.

In conclusion, the present study demonstrated a novel molecular mechanism involving the inhibition of $\beta$-catenin signaling, through which the regulation of Wnt3a secretion by Tmem64 is mediated. Tmem64 may act as a modulator of prostate cancer to inhibit or delay its progression by inhibiting the transcriptional activity of $\beta$-catenin. These findings indicate that Tmem64 has a role in the metastatic progression of prostate cancer to the bone. Further studies on use of Tmem64 as a therapeutic target in prostate cancer bone metastasis in the clinical setting is warranted.

\section{Acknowledgements}

Not applicable.

\section{Funding}

This research was supported by Basic Science Research Program through the National Research Foundation of Korea (NRF) funded by the Ministry of Education, Science and Technology (grant nos. 2012R1A1A2041418 and 2016R1D1A3B03930719).

\section{Availability of data and materials}

All data generated or analyzed during this study are included in this published article.

\section{Authors' contributions}

YHM, WL and BCJ performed the experiments. YHM and WL reviewed, analyzed and interpreted the data. BCJ wrote the paper. All authors discussed the results and commented on the manuscript. YHM, WL and BCJ take responsibility for the integrity of the data analysis. All authors read and approved the final manuscript.

\section{Ethics approval and consent to participate}

All experimental procedures involving animals were performed in compliance with institutional and government requirements and were approved by the Institutional Animal Care and Use Committee (CIACUC2015-A0032), Chosun University, Gwangju, Korea.

\section{Patient consent for publication}

Not applicable.

\section{Competing interests}

The authors declare that they have no competing interests.

\section{References}

1. Ciani L and Salinas PC: WNTs in the vertebrate nervous system: From patterning to neuronal connectivity. Nat Rev Neurosci 6: 351-362, 2005

2. Wang Y, Li YP, Paulson C, Shao JZ, Zhang X, Wu M and Chen W: Wnt and the Wnt signaling pathway in bone development and disease. Front Biosci (Landmark Ed) 19: 379-407, 2014.

3. Regard JB, Zhong Z, Williams BO and Yang Y: Wnt signaling in bone development and disease: Making stronger bone with Wnts. Cold Spring Harb Perspect Biol 4: a007997, 2012.

4. Niemann S, Zhao C, Pascu F, Stahl U, Aulepp U, Niswander L, Weber JL and Müller U: Homozygous WNT3 mutation causes tetra-amelia in a large consanguineous family. Am J Hum Genet 74: 558-563, 2004.

5. Day TF, Guo X, Garrett-Beal L and Yang Y: Wnt/beta-catenin signaling in mesenchymal progenitors controls osteoblast and chondrocyte differentiation during vertebrate skeletogenesis. Dev Cell 8: 739-750, 2005.

6. Rodda SJ and McMahon AP: Distinct roles for Hedgehog and canonical Wnt signaling in specification, differentiation and maintenance of osteoblast progenitors. Development 133: 3231-3244, 2006.

7. Holmen SL, Zylstra CR, Mukherjee A, Sigler RE, Faugere MC, Bouxsein ML, Deng L, Clemens TL and Williams BO: Essential role of beta-catenin in postnatal bone acquisition. $\mathrm{J}$ Biol Chem 280: 21162-21168, 2005.

8. MacDonald BT, Tamai K and He X: Wnt/beta-catenin signaling: Components, mechanisms, and diseases. Dev Cell 17: 9-26, 2009.

9. Li Z, Xu Z, Duan C, Liu W, Sun J and Han B: Role of TCF/LEF transcription factors in bone development and osteogenesis. Int J Med Sci 15: 1415-1422, 2018.

10. Krishnan V, Bryant HU and Macdougald OA: Regulation of bone mass by Wnt signaling. J Clin Invest 116: 1202-1209, 2006.

11. Komiya $\mathrm{Y}$ and Habas R: Wnt signal transduction pathways. Organogenesis 4: 68-75, 2008.

12. Smolich BD, McMahon JA, McMahon AP and Papkoff J: Wnt family proteins are secreted and associated with the cell surface. Mol Biol Cell 4: 1267-1275, 1993. 
13. Komekado $\mathrm{H}$, Yamamoto $\mathrm{H}$, Chiba $\mathrm{T}$ and Kikuchi $\mathrm{A}$ : Glycosylation and palmitoylation of Wnt-3a are coupled to produce an active form of Wnt-3a. Genes Cells 12: 521-534, 2007.

14. Jeong BC, Kim TS, Kim HS, Lee SH and Choi Y: Transmembrane protein 64 reciprocally regulates osteoblast and adipocyte differentiation by modulating $\mathrm{Wnt} / \beta$-catenin signaling. Bone 78 : 165-173, 2015.

15. Lim W, Kim J, Kim S, Karna S, Won J, Jeon SM, Kim SY, Choi Y, Choi $\mathrm{H}$ and Kim O: Modulation of lipopolysaccharide-induced NF- $\kappa$ B signaling pathway by $635 \mathrm{~nm}$ irradiation via heat shock protein 27 in human gingival fibroblast cells. Photochem Photobiol 89: 199-207, 2013.

16. Arguello F, Baggs RB and Frantz CN: A murine model of experimental metastasis to bone and bone marrow. Cancer Res 48: 6876-6881, 1988.

17. He X, Semenov M, Tamai K and Zeng X: LDL receptor-related proteins 5 and 6 in Wnt/beta-catenin signaling: Arrows point the way. Development 131: 1663-1677, 2004.

18. Wang Y, Huang Y, Zhang M, Zhang X, Tang X and Kang Y: Bioinformatic analysis of the possible regulative network of miR-30a/e in Cardiomyocytes 2 Days post myocardial infarction. Acta Cardiol Sin 34: 175-188, 2018.

19. Chu K, Cheng CJ, Ye X, Lee YC, Zurita AJ, Chen DT, Yu-Lee LY, Zhang S, Yeh ET, Hu MC, et al: Cadherin-11 promotes the metastasis of prostate cancer cells to bone. Mol Cancer Res 6 : 1259-1267, 2008.

20. Murillo-Garzón V and Kypta R: WNT signalling in prostate cancer. Nat Rev Urol 14: 683-696, 2017.

21. Nandana S, Tripathi M, Duan P, Chu CY, Mishra R, Liu C, Jin R, Yamashita H, Zayzafoon M, Bhowmick NA, et al: Bone metastasis of prostate cancer can be therapeutically targeted at the TBX2-WNT signaling axis. Cancer Res 77: 1331-1344, 2017.

22. Kim H, Kim T, Jeong BC, Cho IT, Han D, Takegahara N Negishi-Koga T, Takayanagi H, Lee JH, Sul JY, et al: Tmem64 modulates calcium signaling during RANKL-mediated osteoclast differentiation. Cell Metab 17: 249-260, 2013.

23. Verras $M$ and Sun Z: Roles and regulation of Wnt signaling and beta-catenin in prostate cancer. Cancer Lett 237: 22-32, 2006.
24. Yu X, Wang Y, DeGraff DJ, Wills ML and Matusik RJ: Wnt/ $\beta$-catenin activation promotes prostate tumor progression in a mouse model. Oncogene 30: 1868-1879, 2011.

25. Bryden AA, Hoyland JA, Freemont AJ, Clarke NW, Schembri Wismayer D and George NJ: E-cadherin and beta-catenin are down-regulated in prostatic bone metastases. BJU Int 89: 400-403, 2002.

26. Yang Y, Ye YC, Chen Y, Zhao JL, Gao CC, Han H, Liu WC and Qin HY: Crosstalk between hepatic tumor cells and macrophages via Wnt/ $\beta$-catenin signaling promotes M2-like macrophage polarization and reinforces tumor malignant behaviors. Cell Death Dis 9: 793, 2018.

27. Kishida S, Yamamoto H, Ikeda S, Kishida M, Sakamoto I, Koyama $S$ and Kikuchi A: Axin, a negative regulator of the wnt signaling pathway, directly interacts with adenomatous polyposis coli and regulates the stabilization of beta-catenin. J Biol Chem 273: 10823-10826, 1998.

28. Novellasdemunt L, Antas P and Li VS: Targeting Wnt signaling in colorectal cancer. A review in the theme: Cell signaling: Proteins, pathways and mechanisms. Am J Physiol Cell Physiol 309: C511-C521, 2015.

29. Powell SM, Zilz N, Beazer-Barclay Y, Bryan TM, Hamilton SR, Thibodeau SN, Vogelstein B and Kinzler KW: APC mutations occur early during colorectal tumorigenesis. Nature 359: 235-237, 1992.

30. Logan CY and Nusse R: The Wnt signaling pathway in development and disease. Annu Rev Cell Dev Biol 20: 781-810, 2004.

31. Lee HJ, Li J, Vickman RE, Li J, Liu R, Durkes AC, Elzey BD, Yue S, Liu X, Ratliff TL and Cheng JX: Cholesterol esterification inhibition suppresses prostate cancer metastasis by impairing the Wnt/ß-catenin Pathway. Mol Cancer Res 16: 974-985, 2018.

32. Suva LJ, Washam C, Nicholas RW and Griffin RJ: Bone metastasis: Mechanisms and therapeutic opportunities. Nat Rev Endocrinol 7: 208-218, 2011.

This work is licensed under a Creative Commons Attribution-NonCommercial-NoDerivatives 4.0 International (CC BY-NC-ND 4.0) License. 